\title{
We are what we eat: how the diet of infants affects their gut microbiome
}

\author{
Mihai Pop* \\ See research article www.genomebiology.com/2012/13/4/R32
}

\begin{abstract}
Simultaneous analysis of the gut microbiome and host gene expression in infants reveals the impact of diet (breastfeeding versus formula) on host-microbiome interactions.
\end{abstract}

Keywords infant development, gut microbiome, hostmicrobiome interactions, breastfeeding

\section{Bacteria shape our lives}

Childbirth marks a child's sudden transition from the protected intra-uterine environment to the complex world we live in. From the first breath, the newborn starts to learn how to feel, hear, see, grasp, walk, and eventually talk. In other words, infants learn how to adapt to, and interact with the physical world through a succession of cognitive and physical developmental stages. Although this amazing process is familiar to anyone who has ever interacted with a developing child, an equally important developmental process also takes place within the child's gut, mostly unseen, as the body is rapidly populated by microbes. A study by Chapkin and colleagues published in this issue of Genome Biology [1] provides a first insight into the interactions between microbes and the developing infant and how these interactions are affected by diet.

Gastro-intestinal microbes are important for our wellbeing. The gut microbiota is estimated to include $10^{13}$ to $10^{14}$ microbial cells (more than the total number of cells in the human body), and it helps to digest complex starches and provides essential vitamins (such as $\mathrm{K}$ and B12) and other nutrients [2]. Imbalances in the normal gut microbiota have been shown to correlate with a range of diseases, including necrotizing enterocolitis, inflam-

*Correspondence: mpop@umd.edu

Department of Computer Science and Center for Bioinformatics and Computational Biology, University of Maryland, College Park, MD 20742, USA matory bowel syndromes, and even a range of allergic disorders, such as asthma and atopic dermatitis.

In infants, there is strong evidence that the colonization of the body by microbes has an important influence on the development of their immune system [3]. Although the intra-uterine environment, originally believed to be sterile, was recently shown to contain some bacteria [4], the majority of the microbes that will eventually populate the human body are acquired after birth. The mothers' vaginal, gastro-intestinal, and skin bacteria are the first to colonize the newborn infant [5], triggering a succession of colonization stages that, over the period of roughly 1 year, lead to the establishment of an 'adult-like' gastrointestinal flora [6].

Recent studies have started to shed light on the dynamic changes that occur within the gut of infants [5-8]. Early on, the gut becomes colonized by primarily aerobic organisms, such as enterobacteria, staphylococci, and streptococci, many of which have the potential to be pathogenic. These early colonizers begin to change the gut environment, paving the way for colonization by an increasingly anaerobic community of microbes [5]. The structure of the gut community continues to change over the first year of life and thereafter in response to external factors such as diet and antibiotic use [6,8]. Weaning, breastfeeding status, and the successive introduction of different types of food all correspondingly affect the infant gut biome [8]. Also, the mode of delivery (vaginal versus cesarean) influences the population of bacteria that initially colonize the gut [9] and may significantly affect the progression of the microbiota for months after birth [5].

\section{Interactions between infants and their microbiota}

Most of the research on the progression of the gut microbiota in infants has so far focused on the bacteria alone, in part because of the inherent difficulty in assessing the host response. Although stool samples are easy to obtain even from very young infants, examining gene expression within the intestines requires invasive procedures that cannot be justified in the healthy population. As a result, the interaction between microbiome 
and host has previously been explored only in animal or in vitro models.

A new study by Chapkin and colleagues [1] proposes an interesting new approach for non-invasively examining host gene expression within the infant intestinal wall and looking for correlations between this expression and the structure of the gut microbial community. They observed that the human body sheds epithelial cells from the intestines, which can then be recovered from the stool, providing the means to explore the transcriptional activity in the intestinal wall. They used this approach to explore the effect of diet on the host-microbiome interaction in a small cohort of 3-month-old children.

Their study examined stool samples from 12 children, 6 of whom were exclusively breastfed, the remaining being fed a commercial infant formula. From each stool sample, they extracted bacterial DNA, which was subjected to high-throughput sequencing on the Roche/454 platform, and eukaryotic mRNA (from shed epithelial cells), which was analyzed on a microarray platform. The mRNA data had been published earlier by the authors and reanalyzed in the context of the gut microbiota as part of the current study [1].

The taxonomic assignment of bacterial reads confirmed the previously reported impact of diet on the gut microbiome: the microbiota of breastfed and formula-fed infants differed significantly in both composition and diversity [1]. The breastfed samples were more heterogeneous than the formula-fed samples and contained a higher taxonomic diversity (as measured by the ShannonWiener index). This observation may confirm the popularly believed benefit of breastfeeding - in ecological systems greater population diversity is thought to imply greater system stability [10]. Similarly, this insight could guide the development of pro- and pre-biotic supplements to infant formula that are aimed at increasing the bacterial diversity within the gut.

Functional analysis of the gut microbiome revealed a significant enrichment of genes related to microbial virulence in the microbiome of breastfed babies, a result complemented by an enrichment in immunity and defense gene expression in the host transcriptome. This result is perhaps counter-intuitive as it seems to imply a potentially unhealthy gut community. However, the infant gut microbiome has an important role in the development of the host immune system, and potential pathogenic organisms are often the early colonizers of the gut. It is thus likely that these initial attacks on the host are a necessary factor in the development of host immunity and, therefore, the increased virulence in the breastfed infants is beneficial to development.

Combining the microbiome and host response in a multi-variate statistical analysis allowed the authors to further demonstrate the strong correlation between microbiome virulence characteristics and host immune response [1]. This analysis revealed associations between several immunity and mucosal defense genes and the host microbiome. Genes enriched in breastfed infants seemed to demonstrate a closer interaction between microbiome and host in them than in formula-fed infants, further underscoring the potential benefits of breastfeeding.

\section{Breast milk or formula?}

The study by Chapkin and colleagues [1] is an important first step towards better understanding the biological mechanisms underlying the parallel development of host and microbiome during early life. They demonstrate the power of new experimental and analytical approaches that enable the simultaneous analysis of the microbiome and the host response. Such approaches will be critical in developing our understanding of the numerous factors that affect the development of the healthy host-microbiome consortium in human infants.

The authors' analysis of the effects of diet seems to support the commonly held view that breastfeeding has a beneficial role in early life [1]. However, it is important to caution against generalization. The results described here were derived from a small number of children and need to be confirmed in a larger population. More importantly, however, the main contribution of this work is the methodology developed that will enable a much more detailed analysis of the interactions between host and microbiome. This will allow a more nuanced evaluation of all the factors that affect child development, including mode of delivery, breastfeeding status, timing of introduction and composition of the solid food diet, and the many other factors that could have an impact on the development and well-being of children.

Competing interests

The author declares that he has no competing interests.

\section{Acknowledgments}

MP was supported in part by the National Institutes of Health, grant R01HG-004885 and by the William and Melinda Gates Foundation.

Published: 30 April 2012

\section{References}

1. Schwartz S, Friedberg I, Ivanov IV, Davidson LA, Goldsby JS, Dahl DB, Herman D, Wang M, Donovan SM, Chapkin RS: A metagenomic study of dietdependent interaction between gut microflora and host in infants reveals differences in developmental and immune responses. Genome Bio/ 2012

2. Gill SR, Pop M, Deboy RT, Eckburg PB, Turnbaugh PJ, Samuel BS, Gordon II, Relman DA, Fraser-Liggett CM, Nelson KE: Metagenomic analysis of the human distal gut microbiome. Science 2006, 312:1355-1359.

3. Hooper LV: Bacterial contributions to mammalian gut development. Trends Microbiol 2004, 12:129-134.

4. Jimenez E, Marin ML, Martin R, Odriozola JM, Olivares M, Xaus J, Fernandez L, Rodriguez JM: Is meconium from healthy newborns actually sterile? Res Microbio/ 2008, 159:187-193.

5. Morelli L: Postnatal development of intestinal microflora as influenced by 
infant nutrition. J Nutr 2008, 138:1791S-1795S.

6. Palmer C, Bik EM, DiGiulio DB, Relman DA, Brown PO: Development of the human infant intestinal microbiota. PLoS Biol 2007, 5:e177.

7. Morowitz MJ, Denef VJ, Costello EK, Thomas BC, Poroyko V, Relman DA, Banfield JF: Strain-resolved community genomic analysis of gut microbial colonization in a premature infant. Proc Natl Acad SciU S A 2011, 108:1128-1133.

8. Koenig JE, Spor A, Scalfone N, Fricker AD, Stombaugh J, Knight R, Angenent LT, Ley RE: Succession of microbial consortia in the developing infant gut microbiome. Proc Natl Acad Sci U S A 2011, 108 Suppl 1:4578-4585.

9. Dominguez-Bello MG, Costello EK, Contreras M, Magris M, Hidalgo G, Fierer N, Knight R: Delivery mode shapes the acquisition and structure of the initial microbiota across multiple body habitats in newborns. Proc Natl Acad Sci USA 2010, 107:11971-11975.

10. McCann KS: The diversity-stability debate. Nature 2000, 405:228-233.

doi:10.1186/gb-2012-13-4-152

Cite this article as: Pop M: We are what we eat: how the diet of infants

affects their gut microbiome. Genome Biology 2012, 13:152. 